Check for updates

Cite this: RSC Adv., 2017, 7, 47324

Received 10th August 2017 Accepted 2nd October 2017

DOI: $10.1039 / \mathrm{c} 7 \mathrm{ra0} 8857 \mathrm{~d}$

rsc.li/rsc-advances

\title{
A novel triazine-rich polymer wrapped MMT: synthesis, characterization and its application in flame-retardant poly(butylene terephthalate)
}

\begin{abstract}
Fei Xin, (iD) *a Chao Guo, ${ }^{a}$ Yu Chen, ${ }^{b}$ Hailong Zhang ${ }^{a}$ and Lijun Qian (D) ${ }^{a}$
A novel flame retardant (PTAC-MMT) was prepared by wrapping montmorillonite (MMT) with poly $(2,4,6$ triallyloxy-1,3,5-triazine) (TAC) via in situ polymerization, and its structure and properties were characterized by Fourier transformed infrared (FT-IR) spectroscopy, ${ }^{1} \mathrm{H}$ nuclear magnetic resonance $\left({ }^{1} \mathrm{H}\right.$ NMR) and ${ }^{13} \mathrm{C} N M R$, transmission electron microscopy (TEM), X-ray powder diffraction (XRD) and thermogravimetric analysis (TGA) measurements. The PTAC-MMT and aluminum diethylphosphinate (AIPi) were then incorporated into poly(butylene terephthalate) (PBT) to improve the flame retardancy. The sample containing $1.67 \mathrm{wt} \%$ PTAC-MMT and $8.33 \mathrm{wt} \%$ AlPi had a LOI value of $36.4 \%$ and achieved UL94 V-0 classification. The wrapping flame retardants exhibited excellent anti-dripping effect in PBT composite. This work could provide a novel way to prepare modified MMT and the as-prepared flame retardants.
\end{abstract}

\section{Introduction}

Polybutylene terephthalate (PBT) is an important thermoplastic polymer in modern industry, and has been widely used because of its good mechanical properties, chemical resistance, low cost, high processability, and moderate recyclability ${ }^{1-3}$ However, they are flammable and tend to drip during burning, which contributes to fire spread. Therefore, it is a challenging task and has caused great concern to impart both flame retardancy and dripping resistance to $\mathrm{PBT}^{4-7}$ Although several commercially available systems for flame retardancy of PBT consist of a halogen-containing additive and a synergist, they are persistent, bio-accumulative, toxic, and are prohibited under various legislations. ${ }^{8-11}$

To obtain a highly efficient and "safer" flame-retardant PBT, the syntheses of some new organophosphorous compounds or phosphorus-nitrogen compounds and inorganic additives have already been conducted. ${ }^{12-17}$ The improvement of flame retardancy attributed to the formation of network char layer created by decomposition of triazine-rich polymer, which can hinder the heat and mass transport. ${ }^{18-20}$ Montmorillonite (MMT) was frequently used in many polymer nanocomposites owing to its particular nano-layer structure. ${ }^{21-23}$ However, the agglomeration of MMT particles might cause poor dispersion in polymer matrix, which also deteriorates the flame retardancy efficiency and mechanical performances. ${ }^{24-27}$ Therefore, much effort is necessary devoted to improve the dispersibility of the MMT

${ }^{a}$ School of Materials and Mechanical Engineering, Beijing Technology and Business University, Beijing 100048, People's Republic of China. E-mail: xinfei@th.btbu.edu.cn ${ }^{b}$ Beijing Huateng Hightech Co.,Ltd., Beijing 100048, PR China through organo- or nano-modification of MMT. ${ }^{28-30}$ To design an efficient triazine-rich flame-retardant and to study the effect of their wrapped structure on the properties of PBT composites is of great significance for promoting the development of this field. For example, higher flame retardancy obtained and the mechanical properties improved, which can be attributed to the good interfacial adhesion between wrapped MMT and the polymer matrix.

This work mainly aims at the reduction of the agglomeration of MMT layers in PBT nanocomposites, a triazine-rich polymer, poly(2,4,6-triallyloxy-1,3,5-triazine) (PTAC) wrapped on the surface of MMT was designed and synthesized by in situ intercalation polymerization to obtain PTAC-MMT. The PBT composite containing a small amount of PTAC-MMT in combination with AlPi was prepared, and its flammability was characterized. The synergistic flame-retardant effects of PTAC-MMT/ AlPi were also analyzed. This study will focus on exploring a new route for the efficient use of MMT compared with the common organo-modified MMT.

\section{Experimental}

\subsection{Raw materials}

TAC, 2,4,6-triallyloxy-1,3,5-triazine, were supplied by TCI Shanghai Huacheng Industrial Development Corp. Ltd., China. The original montmorillonite (Na-MMT) in this study was provided by Zhejiang Fenghong Clay Corp. Ltd., China. Aluminum diethylphosphinate (AlPi) was obtained from Clariant Chemicals (China) Ltd. Poly(butylene terephthalate) (PBT) was purchased from Nantong Xingchen synthetic material CO. Ltd., China. Unless otherwise indicated, all materials were used as-received. 


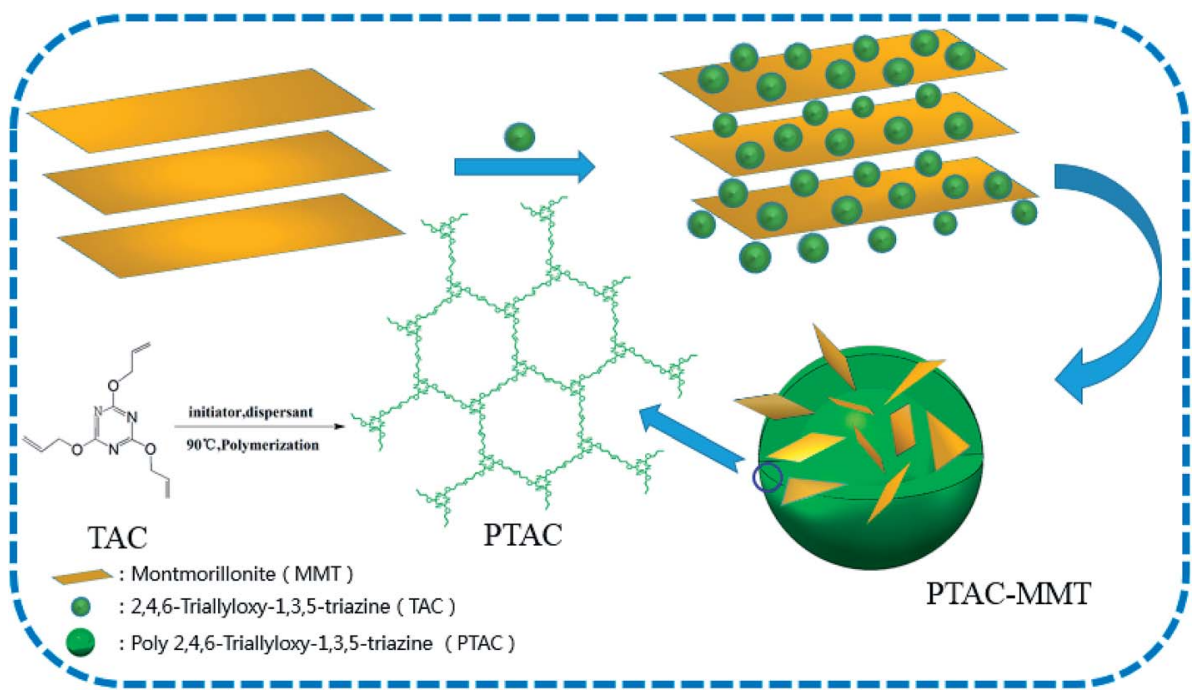

Scheme 1 The schematic illustration of the synthesis procedure of PTAC-MMT and PTAC.

\subsection{Preparation of PTAC-MMT and PTAC}

The wrapped MMT, designated as PTAC-MMT, were prepared as follows. $9.00 \mathrm{~g}$ TAC and $3.00 \mathrm{~g}$ original MMT dissolved in $200 \mathrm{~mL}$ water according to the feed ratio $(3: 1)$ of polymer monomers to MMT and even agitation, then placed in an ultrasonic bath dispersion for $60 \mathrm{~min}$ under $60{ }^{\circ} \mathrm{C}$, inserting TAC in MMT layer using the water solvation effects. Subsequently, the solution were fed into a $500 \mathrm{~mL}$ three-necked flask added initiator and dispersant, then the reaction mixture was maintained under $98{ }^{\circ} \mathrm{C}$ for $3 \mathrm{~h}$ and stir constantly. Finally, resulting emulsion colloid PTAC-MMT was filtered to remove the small molecular weight impurity, and the product was washed at least three times. The resulting product was dried for $6 \mathrm{~h}$ at $110{ }^{\circ} \mathrm{C}$ to a constant weight. Moreover, in order to determine the $C_{\mathrm{PM}}$, a triazine-rich polymer PTAC was synthesized using the same method without the addition of MMT. The schematic representation of preparing process of PTAC-MMT and PTAC are shown in Scheme 1.

\subsection{Preparation of flame-retardant PBT resins}

The compounds of PBT with PTAC-MMT and AlPi were prepared using an LABTECH counter-roating twin-screw extruder with a screw speed of $70 \mathrm{rpm}$. Before mixing, flame retardants were dried at $120{ }^{\circ} \mathrm{C}$ and PBT resin dried at $140{ }^{\circ} \mathrm{C}$ under vacuum for $4 \mathrm{~h}$. The aggregate samples were then transferred to a mold and preheated at $240{ }^{\circ} \mathrm{C}$ for $9 \mathrm{~min}$, then pressed at $10 \mathrm{MPa}$, and cooled to room temperature to obtain the composite sheets. All the details of formulae listed in Table 1.

\subsection{Characterizations}

Fourier transform infrared (FTIR) spectra was detected on a Nicolet iN10MX type spectrometer.

Hydrogen-1 nuclear magnetic resonance $\left({ }^{1} \mathrm{H}\right.$ NMR $)$ and ${ }^{13} \mathrm{C}$ NMR data were obtained using a Bruker $400 \mathrm{MHz}$ WB SolidState NMR Spectrometer.
Table 1 The formulae of the PBT samples ${ }^{a}$

\begin{tabular}{llll}
\hline & \multicolumn{2}{l}{ Composition of formulations in $10 \mathrm{wt} \%$} \\
\cline { 2 - 4 } Samples & PBT (wt $\%)$ & $\begin{array}{l}\text { PTAC-MMT } \\
(\mathrm{wt} \%)\end{array}$ & AlPi (wt\%) \\
\hline Pristine PBT & 100 & 0 & 0 \\
AlPi/PBT & 90 & 0 & 10 \\
1PTAC-MMT/5AlPi/PBT & 90 & 1.67 & 8.33 \\
1PTAC-MMT/4AlPi/PBT & 90 & 2 & 8 \\
1PTAC-MMT/3AlPi/PBT & 90 & 2.5 & 7.5 \\
1PTAC-MMT/2AlPi/PBT & 90 & 3.33 & 6.67 \\
1PTAC-MMT/1AlPi/PBT & 90 & 5 & 5
\end{tabular}

${ }^{a}$ 1PTAC-MMT/5AlPi/PBT means the weight ratio PTAC-MMT to AlPi was $1: 5$.

Morphology of PTAC-MMT were observed with a Talos F200S transmission electron microscope (TEM) at an accelerating voltage of $100 \mathrm{kV}$.

XRD patterns were measured using a RIGAKU Miniflex600 Xray diffractometer with $\mathrm{Cu}$ Ka radiation $(\lambda=0.1541 \mathrm{~nm})$.

Thermo gravimetric analysis (TGA) was recorded on TA instrument Q500 IR thermal gravimetric analyzer with a heating rate of $20{ }^{\circ} \mathrm{C} \min ^{-1}$ from 50 to $700{ }^{\circ} \mathrm{C}$ under $\mathrm{N}_{2}$ atmosphere.

The limiting oxygen index (LOI) values was performed on a FTT (Fire Testing Technology, UK) Dynisco LOI instrument according to ASTM D 2863-97 with sample size of $130 \mathrm{~mm} \times$ $6.5 \mathrm{~mm} \times 3.2 \mathrm{~mm}$.

The vertical burning test (UL-94) were performed on FTT0082 instrument according to ASTM D 3801 testing procedure with sheet dimensions of $130 \mathrm{~mm} \times 13 \mathrm{~mm} \times 3 \mathrm{~mm}$.

The fire behavior was characterized on a FTT cone calorimeter according to ISO5660 under an external heat flux of $50 \mathrm{~kW} \mathrm{~m}{ }^{-2}$ with sample size of $100 \mathrm{~mm} \times 100 \mathrm{~mm} \times 3 \mathrm{~mm}$.

The morphology images of the residues after cone calorimeter test were obtained using a digital camera and Phenom Pro 
field emission scanning electron microscope at high vacuum conditions with a voltage of $5 \mathrm{kV}$.

\section{Results and discussion}

\subsection{Characterization of PTAC-MMT}

Fig. 1 shows the FTIR spectra of TAC, MMT, PTAC and PTACMMT. For MMT, they have few identifiable functional groups. The characteristic absorption bands at $1636 \mathrm{~cm}^{-1}$ and $1038 \mathrm{~cm}^{-1}$, which attributed to $\mathrm{H}-\mathrm{O}-\mathrm{H}$ bending vibration, and $\mathrm{Si}-\mathrm{O}-\mathrm{Si}$ asymmetric stretching vibration, respectively. ${ }^{31}$ Several characteristic peaks appeared in PTAC-MMT after in situ polymerization. In the FTIR spectra of PTAC, it is observed that the strong absorption peaks at $1567 \mathrm{~cm}^{-1}$ are attributed to the vibration of the triazine ring skeleton. The characteristic peaks centered at $1404 \mathrm{~cm}^{-1}$ and $1331 \mathrm{~cm}^{-1}$ correspond to $\mathrm{C}-\mathrm{N}$ and $\mathrm{C}-\mathrm{O}$, respectively. The absorption peak at $931 \mathrm{~cm}^{-1}$ indicates the formation of the $\mathrm{RCH}=\mathrm{CHR}^{\prime}$. Meanwhile, the absorption peak at $1298 \mathrm{~cm}^{-1}$ corresponding to the $\mathrm{RCH}=\mathrm{CH}_{2}$ bond disappear. Therefore, it can be deduced that the PTAC was successfully synthesized. Furthermore, these characteristic peaks in the spectrum of PTAC also appear in the spectrum of PTAC-MMT. It shows that the polymer PTAC exists in PTAC-MMT.

The ${ }^{1} \mathrm{H}$ NMR (a) and ${ }^{13} \mathrm{C}$ NMR (b) spectrum were further used to investigate the chemical structural of PTAC. As shown in Fig. 2, in the ${ }^{1} \mathrm{H}$ solid-state NMR spectrum, it can be found that the ${ }^{1} \mathrm{H}$ of $-\mathrm{CH}_{2}$ from PTAC resonates at $\delta=1.58 \mathrm{ppm},=\mathrm{CH}_{2}$ from PTAC resonates at $\delta=4.69 \mathrm{ppm}$. Another typical peak observed at $\delta=4.06 \mathrm{ppm}$, induced by the proton peak of $\mathrm{RCH}=$ groups. From the ${ }^{13} \mathrm{C}$ solid-state NMR spectrum, the two typical absorption peaks at $\delta=33.41 \mathrm{ppm}$ and $\delta=69.52 \mathrm{ppm}$ were assigned to carbon atoms in the triazine groups and methylene groups, respectively. Furthermore, the two typical absorption peaks at $\delta=118.41 \mathrm{ppm}$ and $\delta=132.59 \mathrm{ppm}$ were assigned to carbon atoms in the $\mathrm{R}_{2} \mathrm{C}=\mathrm{CH}_{2}$ and $\mathrm{RCH}=\mathrm{CHR}^{\prime}$. The results of FTIR and ${ }^{1} \mathrm{H} \mathrm{NMR} /{ }^{13} \mathrm{C}$ NMR clearly verified that PTAC was successfully synthesized.

XRD analysis is a classical method to evaluate whether PTAC was entered into MMT or not, because the interlayer can be estimated by measuring the $d_{001}$ spacing. Fig. 3 shows the XRD

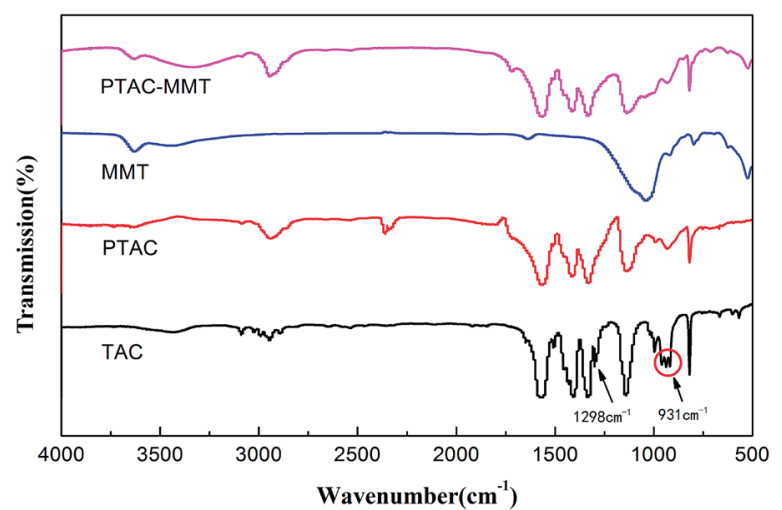

Fig. 1 The FTIR spectra of (a) TAC, (b) PTAC, (c) MMT and (d) PTACMMT.
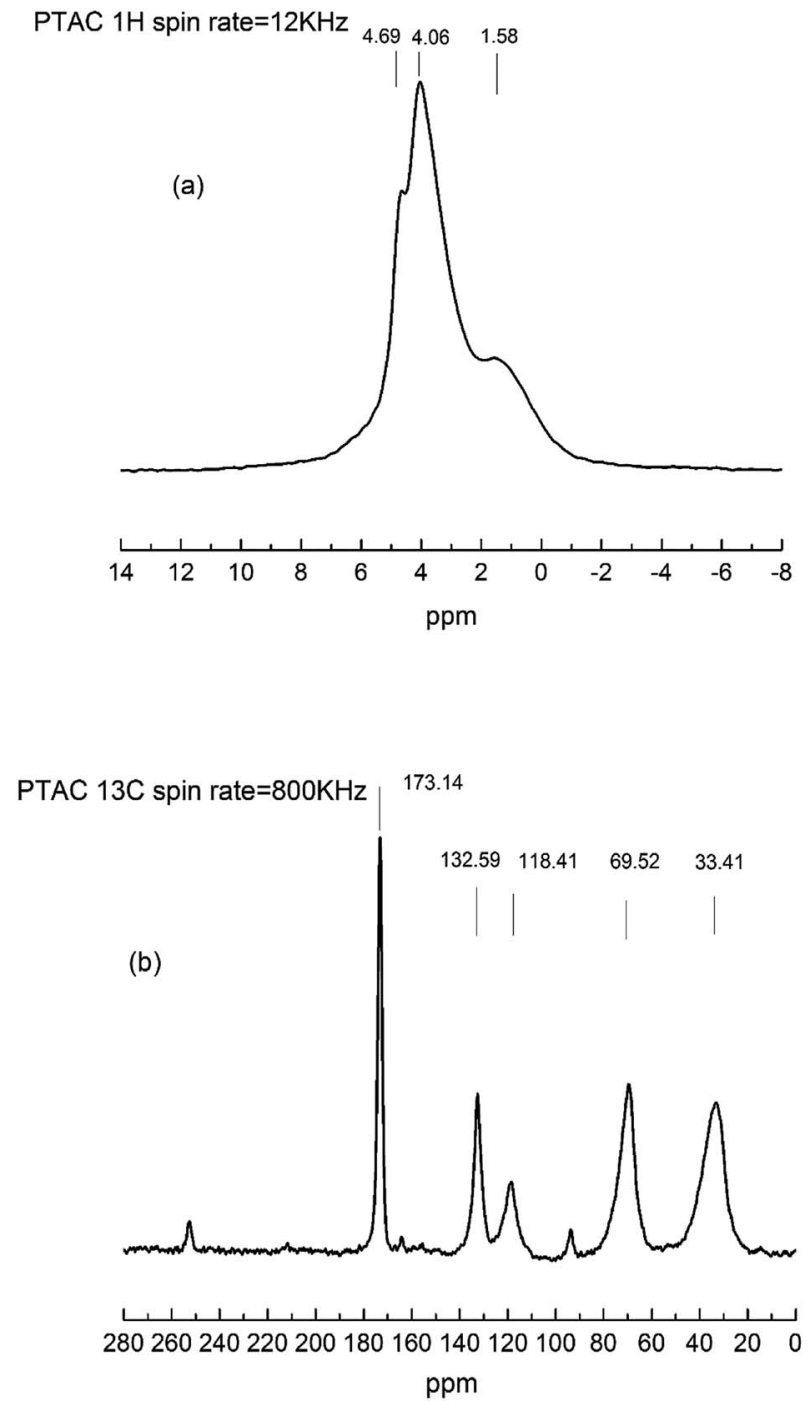

Fig. $2{ }^{1}$ HNMR (a) and ${ }^{13}$ CNMR (b) curves for PTAC

pattern of original MMT and PTAC-MMT, respectively. At $2 \theta=$ $7.19^{\circ}$, there is a strong diffraction peak in MMT. The distance between layers can be calculated according to the Bragg equation.

$$
2 d \sin \theta=n \lambda
$$

where $d$ is the average distance between MMT layers, $\theta$ is the diffraction angle, $\lambda$ is the inlet X-ray wavelength $(0.154 \mathrm{~nm})$, and $n$ is the diffraction number. The calculated distance between MMT layers is $1.23 \mathrm{~nm}$. As can be seen from the figure, the $2 \theta$ value of corresponding XRD peaks decreases with the addition of PTAC into MMT. The layer distance calculated from eqn (1) increases from $1.23 \mathrm{~nm}$ for original MMT to $1.92 \mathrm{~nm}$ for the coated flame retardant with PTAC content of $25 \mathrm{wt} \%$. It suggested that PTAC molecules had entered the silicate layers of MMT.

The TEM images of MMT and PTAC-MMT presented in Fig. 4. One can see a typical TEM of the surface morphology and aqueous suspensions of the original MMT and PTAC-MMT hybrid particles. As shown in Fig. 4(a), silicate lamella of MMT in the forms of aggregation or stack together arbitrary were 


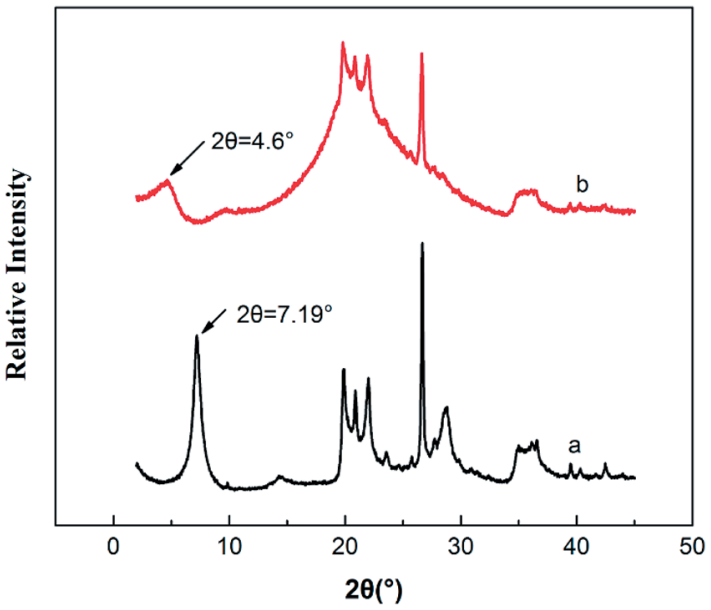

Fig. 3 X-ray diffraction curve of (a) original MMT, (b) PTAC-MMT.
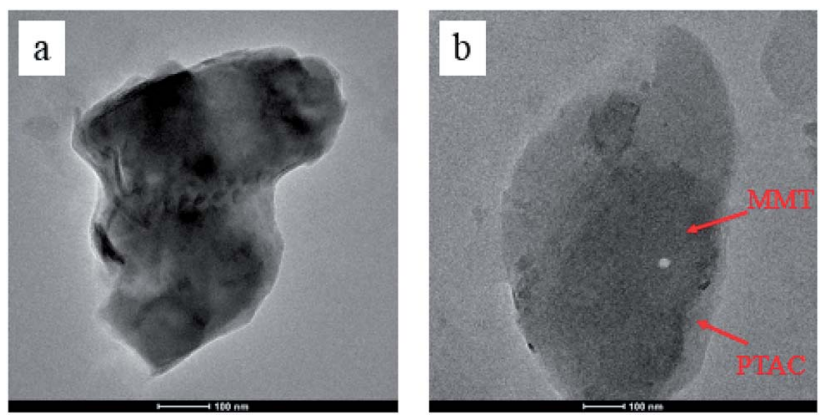

Fig. 4 TEM images of (a) original MMT and (b) PTAC-MMT.

observed. In Fig. 4(b), the bright regions of the photograph at $100 \mathrm{~nm}$ magnification represent the domain polymer of PTAC and the dark lines correspond to the cross section of clay layers. The surface of MMT became obscure and rough because the polymer PTAC wrapped on the external surface of MMT. ${ }^{32}$ These results demonstrated that the PTAC successfully coated onto MMT surface, which was in good agreement with the XRD results. Undoubtedly, the TEM images provide further evidence of the successful wrapping of PTAC-MMT.

Fig. 5 shows the TGA curves of MMT, PTAC, and PTAC-MMT, which were measured from $50{ }^{\circ} \mathrm{C}$ to $700{ }^{\circ} \mathrm{C}$ at $20^{\circ} \mathrm{C} \mathrm{min}{ }^{-1}$ under $\mathrm{N}_{2}$ atmosphere. Obviously, MMT presented good thermal stability under $\mathrm{N}_{2}$ atmosphere. When the temperature was increased up to $700{ }^{\circ} \mathrm{C}$, there was no evident decomposition in MMT, with $96.1 \mathrm{wt} \%$ of residue at that temperature. The synthesized triazine-rich polymer PTAC started to decompose at $318{ }^{\circ} \mathrm{C}$ (weight loss $0.5 \mathrm{wt} \%$ ) and about $8.1 \mathrm{wt} \%$ of residue was left at $700{ }^{\circ} \mathrm{C}$, indicating PTAC had a good char-forming capability. For PTAC-MMT about $23.9 \mathrm{wt} \%$ of residues were left at $700{ }^{\circ} \mathrm{C}$. By comparing the differences in the weight loss between MMT, PTAC, and PTAC-MMT, the content of the polymer PTAC in PTAC-MMT was roughly estimated using the following equation:

$$
W_{\mathrm{PTAC}-\mathrm{MMT}}=\left(1-C_{\mathrm{PM}}\right) \times W_{\mathrm{MMT}}+C_{\mathrm{PM}} \times W_{\mathrm{PTAC}}
$$

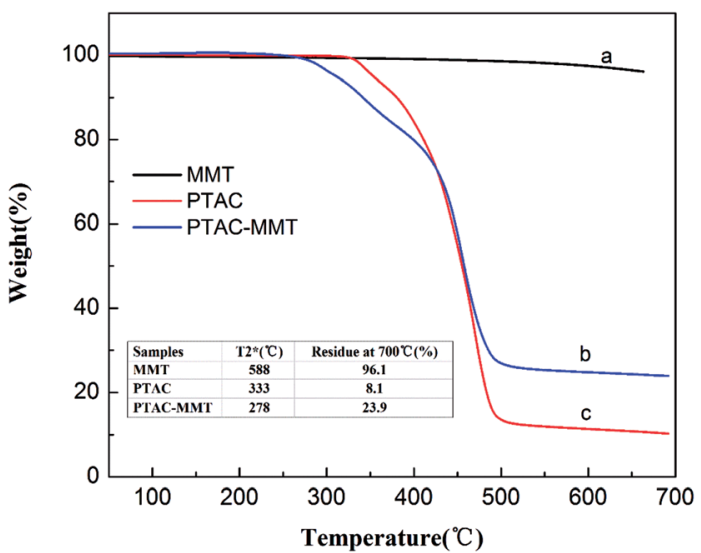

Fig. 5 TGA curves of (a) original MMT, (b) PTAC-MMT and (c) PTAC. $T_{2}^{*}=$ the temperature at which $2 \mathrm{wt} \%$ weight loss takes place.

where $W_{\mathrm{MMT}}, W_{\mathrm{PTAC}}$, and $W_{\mathrm{PTAC}-\mathrm{MMT}}$ are the residues weight fractions of MMT, PTAC, and PTAC-MMT at $700{ }^{\circ} \mathrm{C}$, respectively. $C_{\mathrm{PM}}$, coating rate, denoted the content of the polymer PTAC in PTAC-MMT.

Considering that the mutual influence on thermal degradation between PTAC and MMT is possible, the $C_{\mathrm{PM}}$ is only approximate but not accurate. The calculated $C_{\mathrm{PM}}$ is $82.0 \%$.

\subsection{Structure and morphology of PBT/AlPi/MMT and PBT/ AlPi/PTAC-MMT nanocomposites}

The reinforcement potential of PTAC-MMT is remarkably dependent on the distribution of PTAC-MMT in the PBT matrix. Therefore, it is necessary to determine the dispersibility of PTAC-MMT in the PBT matrix. As shown in Fig. 6, the dispersion of PTAC-MMT was examined by SEM. The fracture surface of pristine PBT in Fig. 6(a) was undefiled without nanofillers. We can see from Fig. 6(b), PTAC-MMT and AlPi particles showed a good dispersion in the PBT and no obvious aggregation was observed. After the introduction of PTAC-MMT, the fracture surface of flame retardant PBT become rough, moreover, it is hard to distinct PBT from PTAC-MMT, indicating a strong interaction between PTAC-MMT and PBT matrix. ${ }^{20}$ This indicates that the PTAC wrapped on the surface of MMT could prevent the agglomeration of MMT and improve the interfacial interactions between PBT and PTAC-MMT.
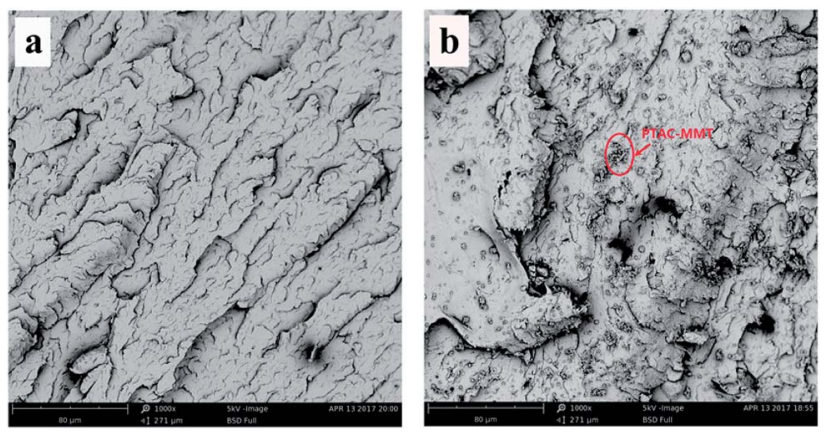

Fig. 6 SEM images of fracture surfaces of (a) pristine PBT $\times 1000$; (b) 10\% 1PTAC-MMT/5AIPi/PBT $\times 1000$. 


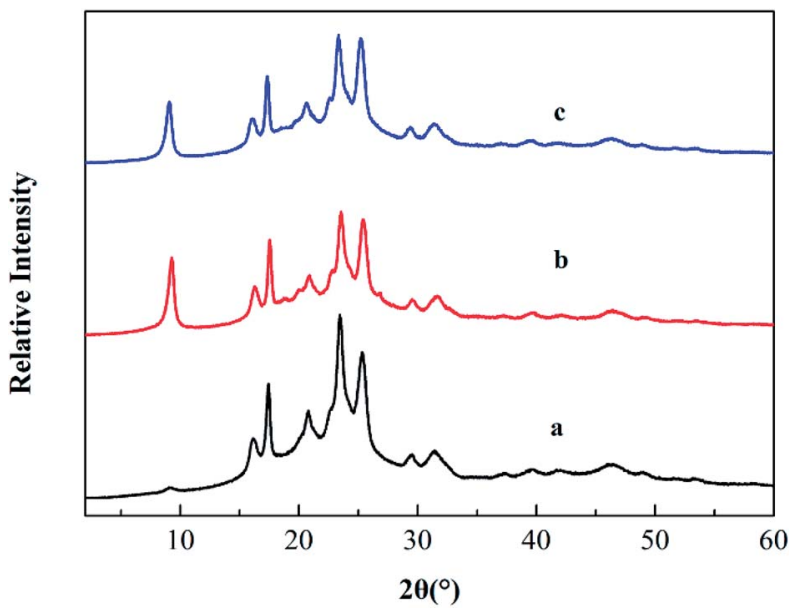

Fig. 7 XRD patterns of (a) PBT, (b) PBT/AIPi/MMT, (c) PBT/AIPi/PTACMMT.

Fig. 7 shows the XRD patterns of PBT, PBT/AlPi/MMT and PBT/AlPi/PTAC-MMT. The PBT/AlPi/MMT mixture did not show any obvious characteristic MMT peaks, whereas the characteristic peaks of PBT were still well retained, which might possibly be related to the dilution effect by the PBT matrix at lower addition of clay. ${ }^{33}$ It can be seen from Fig. 7(c), the diffraction peak of PTAC-MMT at $2 \theta=4.6^{\circ}$ disappeared, which indicated exfoliation of MMT in the polymer matrix. The other peaks of MMT in PTAC-MMT cannot be detected, this implies that the regular and periodic structure of MMT is lost and the MMT layers are exfoliated..$^{23,24}$

\subsection{The flame-retardant performances of the composites}

(a) LOI measurement and UL94 vertical burning tests. The flame retardancy of the PBT composites were detected using LOI and UL94 vertical burning tests, the corresponding results and dripping behavior of all the specimens are presented in Table 2. It can be seen that the presence of AlPi increased the LOI value of PBT effectively, however, it caused dripping. Compared the LOI values of $10 \mathrm{wt} \%$ PTAC-MMT/AlPi flame retardant PBT composites, the LOI firstly increased and then decreased with the increasing of PTAC-MMT. The results can be related with the weight ratio of PTAC-MMT to AlPi in the PBT. The wrapped MMT significantly improved the LOI values of PBT when the mass fractions of PTAC-MMT below $2 \mathrm{wt} \%$. When the mass fraction of PTAC-MMT was only $2 \mathrm{wt} \%$ and $1.67 \mathrm{wt} \%$, the LOI value of PTAC-MMT increased to $35.2 \%$ and $36.4 \%$ respectively. AlPi decomposed mainly through the formation of diethylphosphinic acid in the gas phase, in the presence of triazine-rich polymer and MMT, less phosphorus is released into the gas phase as P-acid, thus, PTAC-MMT promotes its retention as char in the solid phase. ${ }^{34}$ The LOI of PTAC-MMT/ $\mathrm{AlPi} / \mathrm{PBT}$ samples were also higher than those of AlPi/PBT samples when PTAC-MMT below $2.5 \mathrm{wt} \%$ and achieved UL94 V-0 classification, this was due to the synergistic effect of PTACMMT and AlPi on the flame retardancy of PBT. However, when the mass fractions of PTAC-MMT were $5 \mathrm{wt} \%$, the LOI values of
Table 2 LOI and UL94 Results of the PBT Samples

\begin{tabular}{lcccl}
\hline Samples & LOI & Ul-94 & Dripping & $\begin{array}{l}\text { Residue (\%) } \\
\text { at } 700{ }^{\circ} \mathrm{C}\end{array}$ \\
\hline Pristine PBT & 20.4 & NR & Y & 4.83 \\
AlPi/PBT & 34.1 & NR & Y & 7.48 \\
1PTAC-MMT/5AlPi/PBT & 36.4 & V-0 & N & 7.90 \\
1PTAC-MMT/4AlPi/PBT & 35.2 & V-0 & N & 7.82 \\
1PTAC-MMT/3AlPi/PBT & 33.7 & V-0 & N & 7.69 \\
1PTAC-MMT/2AlPi/PBT & 29.3 & V-0 & N & 7.46 \\
1PTAC-MMT/1AlPi/PBT & 27.0 & NR & Y & 7.34
\end{tabular}

Table 3 The data of pristine PBT and flame retardant PBT investigated by the cone calorimeter test ${ }^{a}$

\begin{tabular}{llll}
\hline Samples & $\begin{array}{l}\text { Pristine } \\
\text { PBT }\end{array}$ & $\begin{array}{l}\text { 10\% } \\
\text { AlPi/PBT }\end{array}$ & $\begin{array}{l}\text { 10\% 1PTAC-MMT/ } \\
\text { 5AlPi/PBT }\end{array}$ \\
\hline TTI(s) & 52 & 49 & 41 \\
PHRR & 1648.9 & $714.4(56.6 \%)$ & $661.5(59.8 \%)$ \\
$\left(\mathrm{kW} \mathrm{m}^{-2}\right)$ & & & \\
THR $\left(\mathrm{MJ} \mathrm{m}^{-2}\right)$ & 116.15 & $67.97(41.4 \%)$ & $78.87(32.09 \%)$ \\
av-EHC $\left(\mathrm{MJ} \mathrm{kg}^{-1}\right)$ & 21.26 & $18.96(10.8 \%)$ & $16.32(23.2 \%)$ \\
Char yield (\%) & 4.83 & 7.48 & 7.90
\end{tabular}

${ }^{a}$ The uncertainty is $\pm 10 \%$ of reported the TTI, PHRR, THR, av-EHC and char yield values.

PTAC-MMT/AIPi/PBT only increased from $20.4 \mathrm{wt} \%$ to $27.0 \mathrm{wt} \%$. The addition of AlPi is effective in promoting flame retardance of $\mathrm{PBT}$ in the gas phase, but when the addition of AlPi below $5 \mathrm{wt} \%$, PTAC-MMT in PBT did not result in extinguishing or in preventing flaming dripping and thus got a NR classification in the UL 94 test. In a word, the wrapped MMT is obviously improve flame retardancy of PBT than AlPi and had a good anti-dripping effect when the weight ratio is properly.

(b) Mass loss and cone calorimeter test. Cone calorimeter test is a fire testing apparatus based on the oxygen consumption principle and provides a wealth of information on fire properties and flame retardancy mechanisms of materials. ${ }^{35,36}$ The

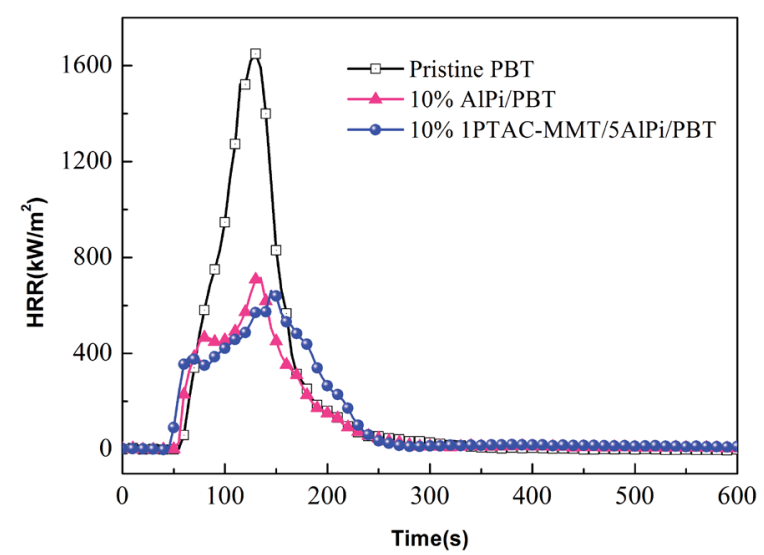

Fig. 8 The HRR curves of pristine PBT, 10\% AIPi/PBT and 10\% 1PTACMMT/5AIPi/PBT. 


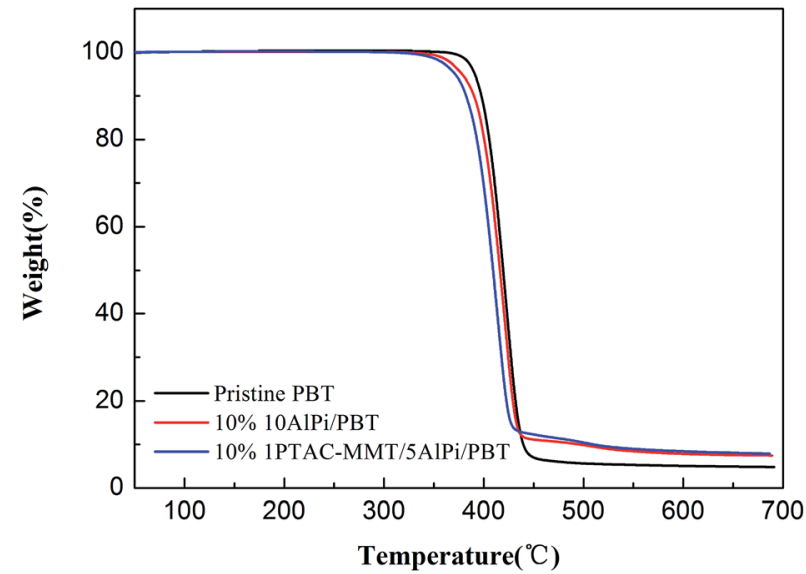

Fig. 9 Normalized mass loss curves of pristine PBT, 10\% AIPi/PBT and $10 \%$ 1PTAC-MMT/5AIPi/PBT from the TGA under $\mathrm{N}_{2}$ atmosphere.

partial characteristic parameters of samples, such as peak of heat release rate (PHRR), total heat release (THR), average effective combustion heat (av-EHC), the time to ignition (TTI), average yield of $\mathrm{CO}(\mathrm{av}-\mathrm{CO} \mathrm{Y})$, average yield of $\mathrm{CO}_{2}\left(\mathrm{av}-\mathrm{CO}_{2} \mathrm{Y}\right)$ and the chsar yield, are shown in Table 3 and Fig. 8 and 9.

As shown in Fig. 8, the HRR curves of pristine PBT had a small shoulder after ignition and before PHRR and represents non-charring materials. ${ }^{37}$ The HRR curve of 10\% 1PTAC-MMT/ 5AlPi/PBT showed a gentle rise after strong initial increase up to the occurring of PHRR. This was due to the 10\% 1PTAC-MMT/ $5 \mathrm{AlPi} / \mathrm{PBT}$ formed a thicker intumescent char, which showed effective barrier effect. A reduction of ignition time was observed for the $10 \%$ PTAC-MMT/AlPi/PBT nanocomposite, we can deduced that PTAC-MMT may interact with AlPi and promote the decomposition of AlPi, the nanocomposite materials is easier to be ignited and release heat.

The quantitative assessment of flame retardancy can precisely compare the difference between AlPi and PTAC-MMT in flame retardant effect. The gas-phase effect and charring are quantified by the reduction of the effective heat of combustion of the volatiles (av-EHC) and the increase in residue, respectively. The change of PHRR is attributed to the changes of the effective heat of combustion, the amount of released fuel and the barrier effect of the char. ${ }^{38,39}$
In Table 3, the av-EHC of $10 \%$ AlPi/PBT was reduced to $89.2 \%$ compared with that of the pristine PBT. The residue increased from $4.83 \%$ to $7.48 \%$, thus the amount of released fuel was reduced to $97.2 \%[(1-7.48 \%) /(1-4.83 \%)]$. The PHRR was reduced to $43.3 \%$. This result demonstrates that the gasphase effect plays a main role in the flame retardancy of $10 \%$ AlPi/PBT. The method also used for calculation of 1PTAC-MMT/ 5AlPi/PBT. The av-EHC was reduced to $76.7 \%$ due to the carbonication effect of PTAC-MMT. The residue increased to $7.90 \%$, thus the amount of released fuel was reduced to $96.8 \%$. The PHRR was reduced to $40.1 \%$. The additional reduction of the PHRR comes from the barrier effect of the intumescent char. It shows that the barrier effect of the carbonaceous char is significant for PTAC-MMT/AlPi/PBT.

Numbers in parenthesis represent the percent reduction.

The increased char formation was observed and the evidence described in the TGA results. Fig. 9 shows the mass loss curves of the pristine $\mathrm{PBT}, 10 \% \mathrm{AlPi} / \mathrm{PBT}$ and 10\% 1PTAC-MMT/5AlPi/PBT. The mass loss curve characterizes the reduction of mass of the material during decomposition. At the end of combustion $\left(700{ }^{\circ} \mathrm{C}\right)$, the char yield of $1 \mathrm{PTAC}-\mathrm{MMT} / 5 \mathrm{AlPi} / \mathrm{PBT}$ was obviously more than those of pristine PBT and 10\% AlPi/PBT. The amount of solid residue collected at the end of decomposition corresponds approximately to the amount of inorganic aluminum phosphates expected from AlPi decomposition and additional carbonaceous char. Therefore, the wrapped MMT present better charring.

The differences of flame retardancy mechanisms of $10 \% \mathrm{AlPi} /$ PBT and 10\% 1PTAC-MMT/5AlPi/PBT are further compared. In condensed phase, the charring and barrier effects of 10\% 1PTACMMT/5AlPi/PBT are stronger than $10 \%$ AlPi/PBT, which just accords with the characteristics of char yield in Fig. 9. The reason is deduced that the interaction of the products from the pyrolysis of the triazine-rich polymer and wrapped MMT promoted charring of the PBT matrix and enhanced the barrier effect of the char. Thus, the reinforced char of $1 \mathrm{PTAC}-\mathrm{MMT} / 5 \mathrm{AlPi} / \mathrm{PBT}$ is more effective to hinder the heat and mass transport compared with that of $10 \%$ AlPi/PBT. The further evidences in detail will be provided in the following discussion.

(c) Morphology analysis of residues. The macroscopic and microscopic morphologies of residues of the pristine PBT, $10 \%$ AlPi/PBT and 10\% 1PTAC-MMT/5AlPi/PBT were further investigated using a digital camera and SEM. The macro-morphology
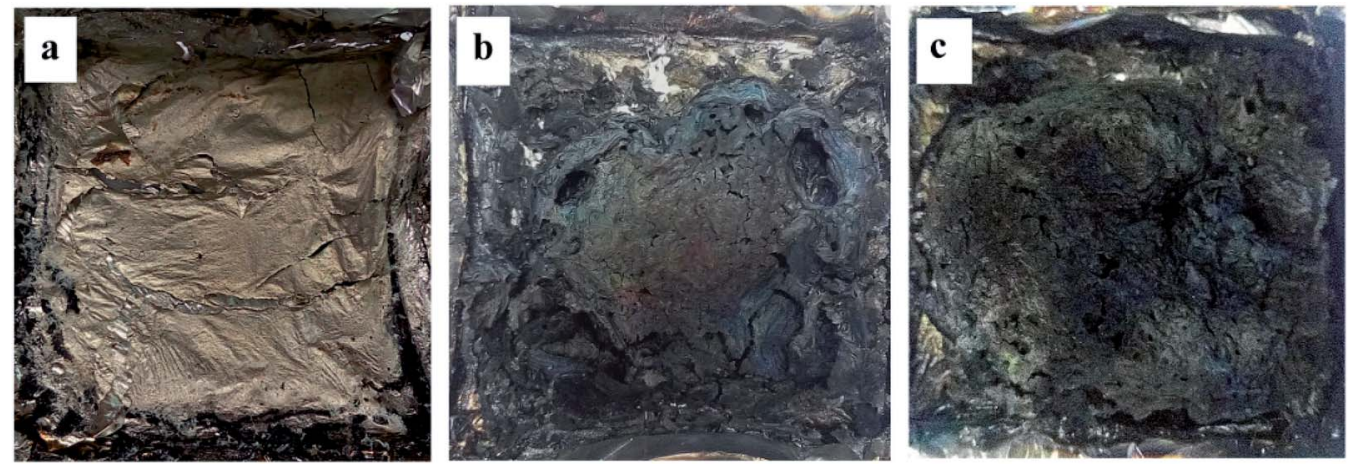

Fig. 10 Digital photos of cone calorimeter residues of (a) pristine PBT; (b) 10\% AIPi/PBT; (c) 10\% 1PTAC-MMT/5AIPi/PBT. 

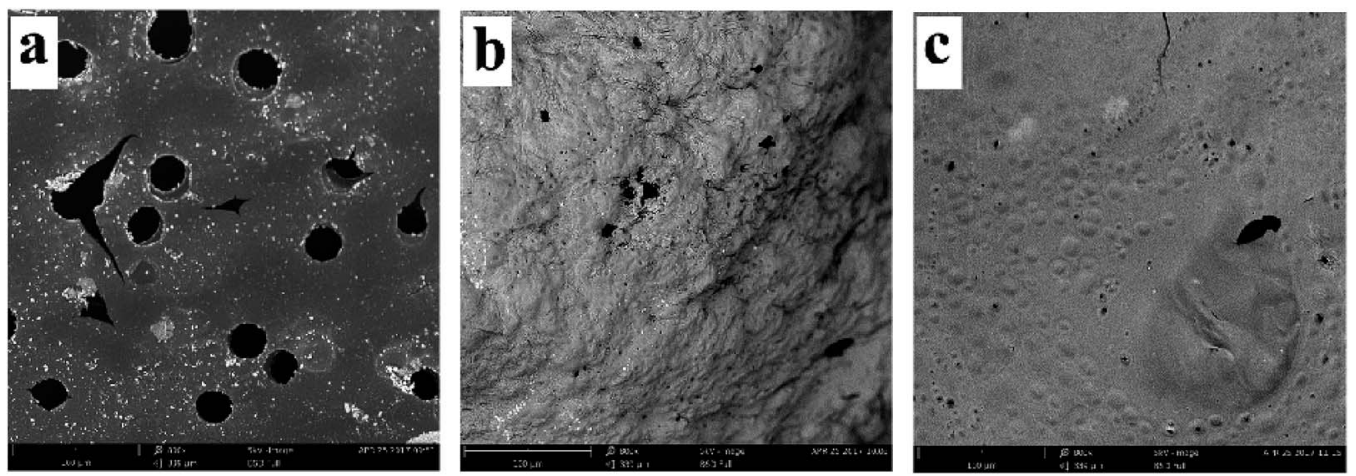

Fig. 11 SEM images analysis of cone calorimeter residues of (a) pristine PBT $\times 800$; (b) $10 \%$ AIPi/PBT $\times 800$; (c) $10 \% 1 \mathrm{PTAC}-\mathrm{MMT} / 5 \mathrm{AlPi} / \mathrm{PBT} \times$ 800.

images of the residues after the cone calorimeter test are shown in Fig. 10. As shown in Fig. 10(a), the residue from pristine PBT sample reserved only tiny amounts of badly broken char, which means that pristine PBT almost totally decomposed during combustion. The residue of $10 \%$ AlPi/PBT in Fig. 10(b) presented the local destruction, and the expansion ratio was not obvious. However, in Fig. 10(c), the residue of 10\% 1PTACMMT/5AlPi/PBT was compact and complete, a preferable intumescent char, which not only effectively hampered the heat and mass transfer but also increased the char yield.

In order to further explore the charring and barrier effects of $10 \%$ 1PTAC-MMT/5AIPi/PBT, SEM images of cone calorimeter residues are shown in Fig. 11. From direct observations, the residue of pristine PBT in Fig. 11(a) had a lot of open holes with different sizes, which provided channels for the combustible volatiles from the inner matrix and heat feedback from the flame. In Fig. 11(b), the residue of $10 \%$ AlPi/PBT appeared exterior osteoporosis and porous because the undispersed network char layer created by decomposition of AlPi was brittle and easy to be damaged. Obviously, the residue of $10 \%$ 1PTACMMT/5AlPi/PBT in Fig. 11(c) was very dense, and few open holes were found. This can be explained by the facts that, on one hand, the wrapped MMT formed a continuous network structured protective layer; on the other hand, the polymer PTAC in PTAC-MMT generated some products to fill the gap of the network char layer of MMT to make it denser. Therefore, this residue structure can effectively seal the combustible volatiles in the matrix and decrease the combustion intensity.

\section{Conclusions}

A novel triazine-rich polymer wrapped MMT, PTAC-MMT, have been synthesized and characterized by FTIR and ${ }^{1} \mathrm{H} \mathrm{NMR} /{ }^{13} \mathrm{C}$ NMR, XRD and TEM. The novel flame retardant have matchable thermal stability with PBT matrix. The combination of PTACMMT and AlPi shows outstanding flame-retardant effect, in particular anti-dripping property in the UL94 test. The sample containing $1.67 \mathrm{wt} \%$ PTAC-MMT and $8.33 \mathrm{wt} \%$ AlPi had a LOI value of $36.4 \%$ and achieved UL94 V-0 classification without any dripping. The wrapped MMT can significantly improve flame retardancy of PBT, and has a good anti-dripping effect. It is proposed that the wrapping with this triazine-rich flame retardant can improve the dispersibility of the MMT and hence enhance the flame retardancy simultaneously.

\section{Conflicts of interest}

There are no conflicts to declare.

\section{Acknowledgements}

The work was supported by the National Natural Science Foundation of China (No. 51403007).

\section{References}

1 S. Sullalti, M. Colonna, C. Berti, M. Fiorini and S. Karanam, Polym. Degrad. Stab., 2012, 97, 566-572.

2 T. Koppl, S. Brehme, D. Pospiech, O. Fischer, F. W. Fabris, V. Altstadt, B. Schartel and M. Doring, J. Appl. Polym. Sci., 2013, 128, 3315-3324.

3 D. Wang, Q. J. Zhang, K. Q. Zhou, W. Yang, Y. Hu and X. L. Gong, J. Hazard. Mater., 2014, 278, 391-400.

4 Y. X. Qi, W. H. Wu, X. W. Liu, H. Q. Qu and J. Z. Xu, Fire Mater., 2017, 41, 195-208.

5 S. V. Levchik and E. D. Weil, Polym. Adv. Technol., 2004, 15, 691-700.

6 S. V. Levchik and E. D. Weil, Polym. Int., 2005, 54, 11-35.

7 E. Gallo, B. Schartel, U. Braun, P. Russo and D. Acierno, Polym. Adv. Technol., 2011, 22, 2382-2391.

8 P. Y. Jia, L. H. Hu, G. D. Feng, C. Y. Bo, J. Zhou, M. Zhang and Y. H. Zhou, RSC Adv., 2017, 7, 897-903.

9 D. Y. Zhu, J. W. Guo, J. X. Xian and S. Q. Fu, $R S C A d v .$, 2017, 7, 39270-39278.

10 A. Salamova, M. H. Hermanson and R. A. Hites, Environ. Sci. Technol., 2014, 48, 6133-6140.

11 P. Gramatica, S. Cassani and A. Sangion, J. Hazard. Mater., 2016, 306, 237-246.

12 P. Liu, M. M. Liu, C. Gao, F. Wang, Y. F. Ding, B. Wen, S. M. Zhang and M. S. Yang, J. Appl. Polym. Sci., 2013, 130, 1301-1307. 
13 R. Sablong, R. Duchateau, C. E. Koning, D. Pospiech, A. Korwitz, H. Komber, S. Starke, L. Häußler, D. Jehnichen and M. A. der Landwehr, Polym. Degrad. Stab., 2011, 96, 334-341.

14 W. H. Wu, S. F. Lv, X. Liu, H. Q. Qu, H. C. Zhang and J. Z. Xu, J. Therm. Anal. Calorim., 2014, 118, 1569-1575.

15 L. Y. Zou, J. Y Liu, X. Q. Liu, X. M. Wang and J. Chen, J. Therm. Anal. Calorim., 2016, 124, 1399-1409.

16 E. Gallo, U. Braun, B. Schartel, P. Russo and D. Acierno, Polym. Degrad. Stab., 2009, 94, 1245-1253.

17 W. Yang, Y. C. Kan, L. Song, Y. Hu, H. D. Lu and R. K. K. Yuen, Polym. Adv. Technol., 2011, 22, 2564-2570.

18 Y. Qiu, L. J. Qian, W. Xi and X. X. Liu, J. Appl. Polym. Sci., 2016, 43241, 1-8.

19 Y. J. Chen, W. Wang, Z. Q. Liu, Y. Y. Yao and L. J. Qian, J. Appl. Polym. Sci., 2016, 44660, 1-8.

20 M. L. Xu, Y. J. Chen, L. J. Qian, J. Y. Wang and S. Tang, J. Appl. Polym. Sci., 2014, 41006, 1-8.

21 Y. M. Wang, J. P. Gao, Y. Q. Ma and U. S. Agarwal, Composites, Part B, 2006, 37, 399-407.

22 Y. H. Yang, Y. C. Li, J. Shields and R. D. Davis, J. Appl. Polym. Sci., 2015, 41767, 1-8.

23 D. Gao, R. Li, B. Lv, J. Z. Ma, F. Tian and J. Zhang, Composites, Part B, 2015, 77, 329-337.

24 G. B. Huang, J. R. Gao, Y. J. Li, L. Han and X. Wang, Polym. Degrad. Stab., 2010, 95, 245-253.

25 S. T. Bee, A. Hassan, C. T. Ratnam, T. T. Tee, L. T. Sin and D. Hui, Composites, Part B, 2014, 61, 41-48.

26 G. Sanchez-Olivares, A. Sanchez-Solis, F. Calderas, L. Medina-Torres, O. Manero, A. Di Blasio and J. Alongi, Appl. Clay Sci., 2014, 102, 254-260.
27 J. C. Liu, M. Y. Fu, M. M. Jing and Q. Y. Li, Polym. Adv. Technol., 2013, 24, 273-281.

28 A. K. Kalkar, V. D. Deshpande and B. S. Vatsaraj, Thermochim. Acta, 2013, 568, 74-94.

29 S. S. Hwang, S. P. Liu, P. P. Hsu, J. M. Yeh, K. C. Chang and Y. Z. Lai, Int. Commun. Heat Mass Transfer, 2010, 37, 10361043.

30 S. J. Wang, F. Xin, Y. Chen, L. J. Qian and Y. J. Chen, Polym. Degrad. Stab., 2016, 129, 133-141.

31 G. G. Chen, X. M. Qi, Y. Guan, F. Peng, C. L. Yao and R. C. Sun, ACS Sustainable Chem. Eng., 2016, 4, 1985-1993.

32 K. C. Chang, G. W. Jang, C. W. Peng, C. Y. Lin, J. C. Shieh, J. M. Yeh, J. C. Yang and W. T. Li, Electrochim. Acta, 2007, 52, 5191-5200.

33 Z. Wang, H. J. Kang, W. Zhang, S. F. Zhang and J. Z. Li, Appl. Surf. Sci., 2017, 401, 271-282.

34 L. Ye, J. Ren, S. Y. Cai, Z. G. Wang and J. B. Li, Chin. J. Polym. Sci., 2016, 34, 785-796.

35 P. Luangtriratana, B. K. Kandola and P. Myler, Mater. Des., 2015, 68, 232-244.

36 X. M. Zhao, D. Xiao, J. P. Alonso and D. Y. Wang, Mater. Des., 2017, 114, 623-632.

37 M. Shabanian, M. Hajibeygi, K. Hedayati, M. Khaleghi and H. A. Khonakdar, Mater. Des., 2016, 110, 811-820.

38 S. Brehme, B. Schartel, J. Goebbels, O. Fischer, D. Pospiech, Y. Bykov and M. Döring, Polym. Degrad. Stab., 2011, 96, 875884.

39 A. Viretto, R. Sonnier, A. Taguet, B. Otazaghine, L. Ferry, J. M. Lopez-Cuesta and C. Lagrève, Fire Mater., 2016, 40, 445-463. 